\title{
The Hannover thermal noise experiment
}

\author{
V Leonhardt, L Ribichini, H Lück and K Danzmann \\ Max-Planck-Institute for Gravitational Physics (Albert-Einstein-Institute) and University of \\ Hannover, Callinstr. 38, D-30167 Hannover, Germany
}

Received 28 August 2003

Published 12 February 2004

Online at stacks.iop.org/CQG/21/S1127 (DOI: 10.1088/0264-9381/21/5/110)

\begin{abstract}
To analyse the thermal noise of the pendulum mode of a suspended mirror, we interferometrically detect the differential movement of two mirrors suspended as multiple-stage pendulums. We present the set-up of this experiment and the current sensitivity, and also the different steps that we took in the past to increase the sensitivity, which include an auto alignment of the laser beam into the resonator eigenmode, changes of the seismic isolation system to more damping stages and higher moments of inertia and an intensive noise hunting.
\end{abstract}

PACS numbers: $95.55 . \mathrm{Ym}, 04.80 . \mathrm{Nn}$

\section{Introduction}

Thermal noise away from the resonances will be a dominating noise source in the gravitational wave interferometers currently starting operation as soon as they reach their designed sensitivity. To distinguish between different existing models for the off-resonant thermal noise and to test different materials, many groups have built dedicated experiments [1-4]. However, a measurement of the off-resonant thermal motion of the pendulum mode of a high- $Q$ pendulum still needs to be done.

In the Hannover thermal noise experiment [5] we have undertaken several steps in the past trying to increase the sensitivity, and several noise sources were investigated. However, the sensitivity is not yet good enough to reach the thermal noise level.

\section{Experiment}

To detect very small differential length changes of our suspended resonator, the frequency of the laser has to be stabilized to a reference resonator with the Pound-Drever-Hall scheme. As an actuator for this servo, we use a piezo attached to the laser crystal and an EOM for high frequencies. A unity gain frequency above $200 \mathrm{kHz}$ is achieved with this scheme. The optical path length inside the reference resonator is $427 \mathrm{~mm}$. It is a ring cavity with three mirrors that are separated by an ULE spacer with a thermal expansion coefficient of $3 \times 10^{-9} \mathrm{~K}^{-1}$. The finesse of the reference resonator is 58 000. The length of the suspended resonator is stabilized to the laser frequency (see figure 1). As an actuator for this lock we use coils acting 


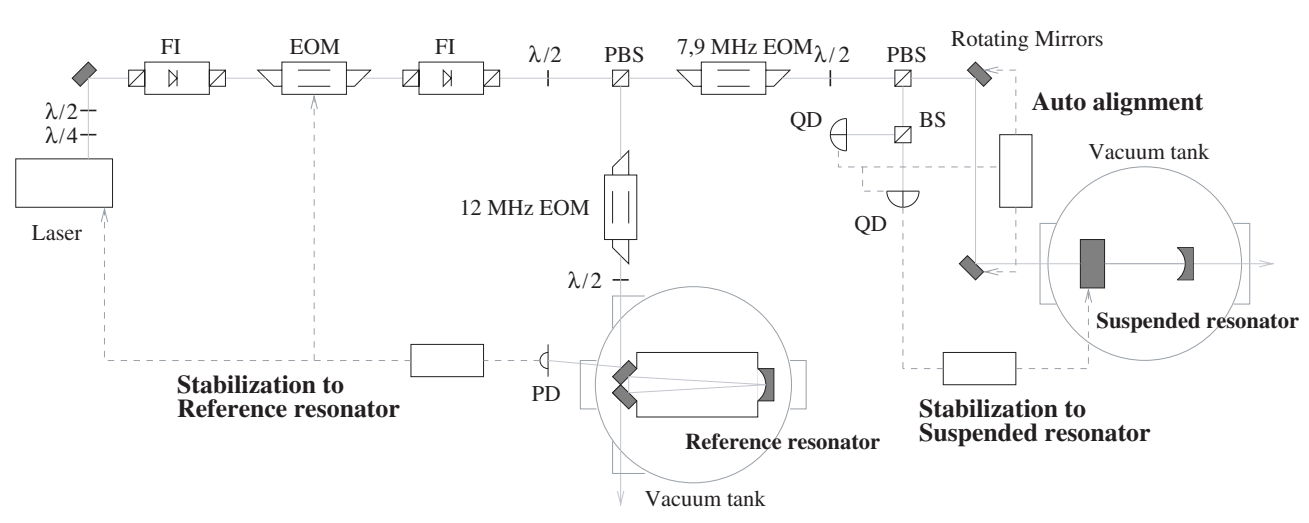

Figure 1. The laser frequency is stabilized to a reference resonator and the beam is centred on the mode of the suspended resonator by an auto alignment system. The length of this cavity is stabilized to the frequency of the laser using a piezo.

on magnets glued to one of the suspended mirrors. These coils have to move the whole mirror and can excite its internal resonances. Recently we used a different actuator, a piezo that only moves a small mirror against a much heavier aluminium mass, to reduce excitations of the large mass. Below the unity gain frequency of a few hundred hertz, the feedback signal of this lock is proportional to the differential movement of the suspended mirrors, and above unity gain the error signal is monitored. To calibrate these signals, a known disturbance can be added to the laser frequency when it is not locked to the reference cavity and the effect of the disturbance can be monitored.

To achieve a constant alignment of the laser light with respect to the eigenmode of the suspended resonator, we have included an auto alignment system. This consists of two quadrant cameras placed with lenses in the near- and far-field of the cavity and makes use of the fact that the light is phase modulated. Separate demodulations and different linear combinations of the diode quadrants allow us to obtain separate signals for the lateral shift and the angle of the laser light against the resonator mode. These signals are then applied to four mirrors mounted on galvanometer scanners. These scanners have their first internal resonances at $310 \mathrm{~Hz}$ and $4 \mathrm{kHz}$ which allowed us a unity gain frequency for the different auto alignment channels between these resonances. In figure 2 the suppression of disturbances at the error point of the vertical shift motion is shown.

The mirrors are currently suspended from four pendulum stages (see figure 3). The first two are identical for both pendulums and include blades for vertical seismic isolation. Suspended from the first two pendulum stages is a frame structure, which is damped by a shadow-sensor-coil-magnet system for all six degrees of freedom. It also houses the coils for damping the pendulum resonances. The two mirrors are suspended as double pendulums from this frame structure. The seismic excitation is further reduced by an active seismic isolation system placed below the vacuum chamber. The two mirrors form a $3 \mathrm{~cm}$ long optical cavity where the first mirror is flat and has a mass of $800 \mathrm{~g}$, and the second one has a radius of curvature of $1 \mathrm{~m}$ and a mass of $50 \mathrm{~g}$.

\section{Results}

The sensitivity in measuring the differential pendulum movement is limited below $50 \mathrm{~Hz}$ by a combination of seismic noise and feedback noise of the control loops damping the pendulum 


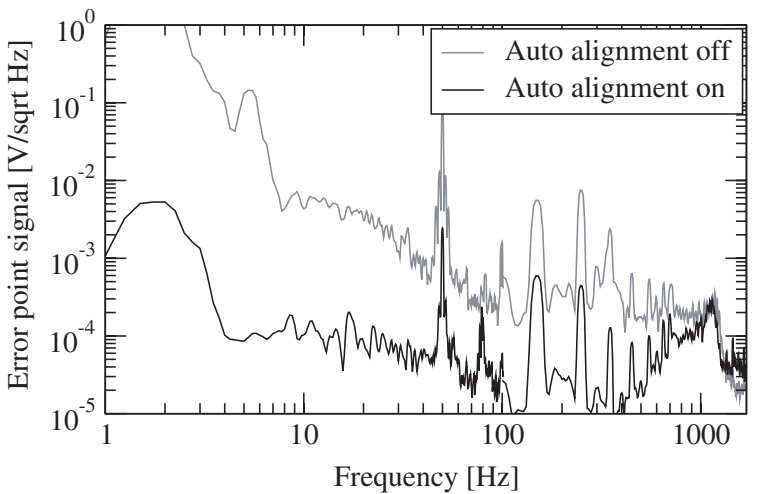

Figure 2. Suppression of movements of the laser beam against the resonator mode in one of the four auto alignment channels (vertical shift), as measured at the error point.

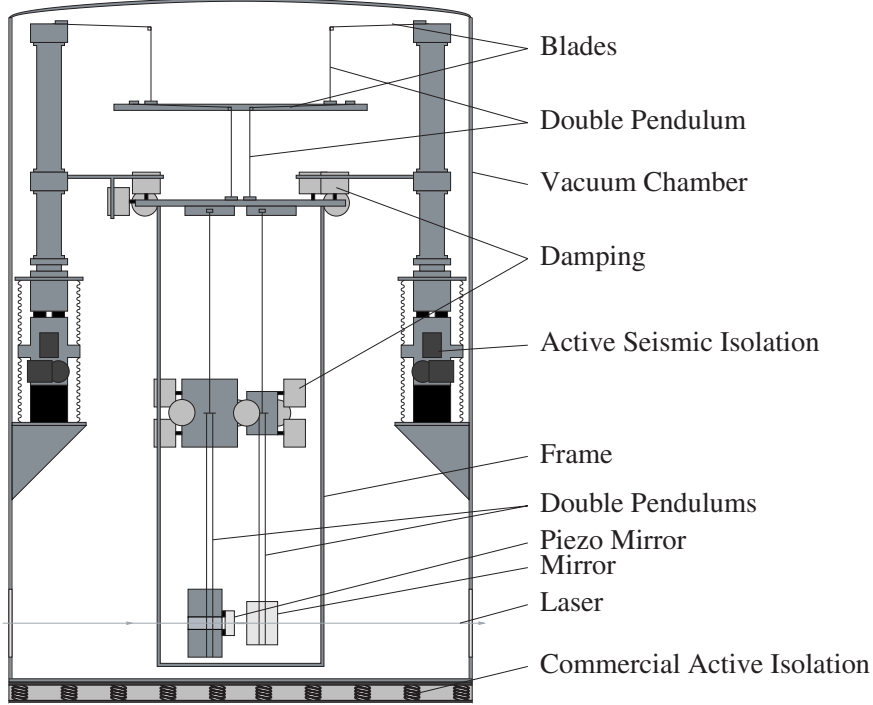

Figure 3. The seismic isolation of the suspended Fabry Perot interferometer includes four pendulum stages and two blade stages and also an active seismic isolation.

resonances. The seismic noise around $10 \mathrm{~Hz}$ is not limited by longitudinal excitation, but by coupling of the other degrees of freedom into longitudinal mirror movement. To minimize the influence of the other degrees of freedom, we have increased the moments of inertia of the pendulum masses by adding small T-shaped arms with heavy masses at the end. This reduced some resonance frequencies and enhanced the sensitivity (see figure 4). However, another attempt with even larger arms attached to the pendulum stages was unsuccessful as the number and amplitude of internal resonances greatly increased.

To detect up to which frequency the sensitivity is limited by pendulum movement, we suspended a fixed cavity to the pendulum stages instead of the two separate mirrors and we locked the length of this cavity to the laser frequency. This eliminated the influence of seismic and other noises moving the mirrors against each other, but kept all other electronic and optical noises. The result was that differential mirror movement dominates the sensitivity up to $50 \mathrm{~Hz}$ (see figure 5). By using two suspended mirrors with a transmission of only 100 ppm instead 


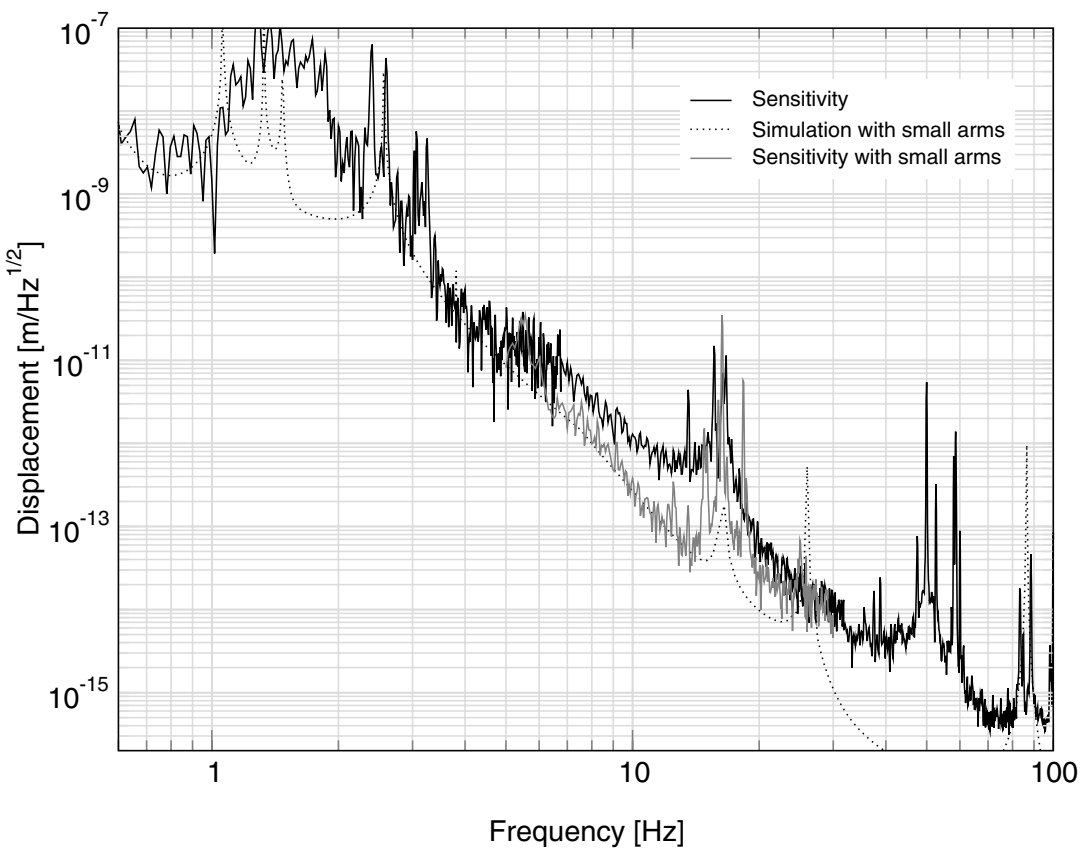

Figure 4. Differential movement of the two suspended mirrors. Increasing the moment of inertia enhanced the sensitivity between $10 \mathrm{~Hz}$ and $20 \mathrm{~Hz}$.

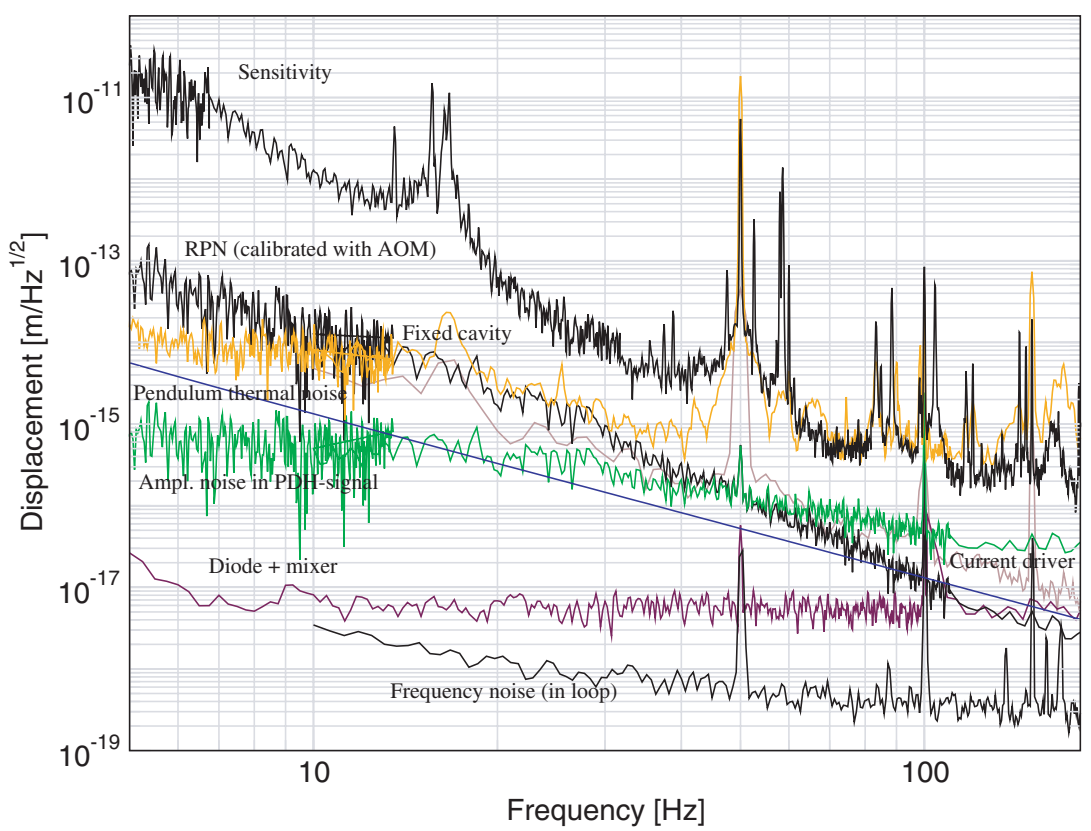

Figure 5. Comparison of different noise sources that might have an influence above $50 \mathrm{~Hz}$, while at lower frequencies seismic noise and noise of the damping of the pendulums dominate. For comparison the predicted thermal noise for viscous damping $\left(Q=10^{6}\right)$ is also shown.

(This figure is in colour only in the electronic version) 


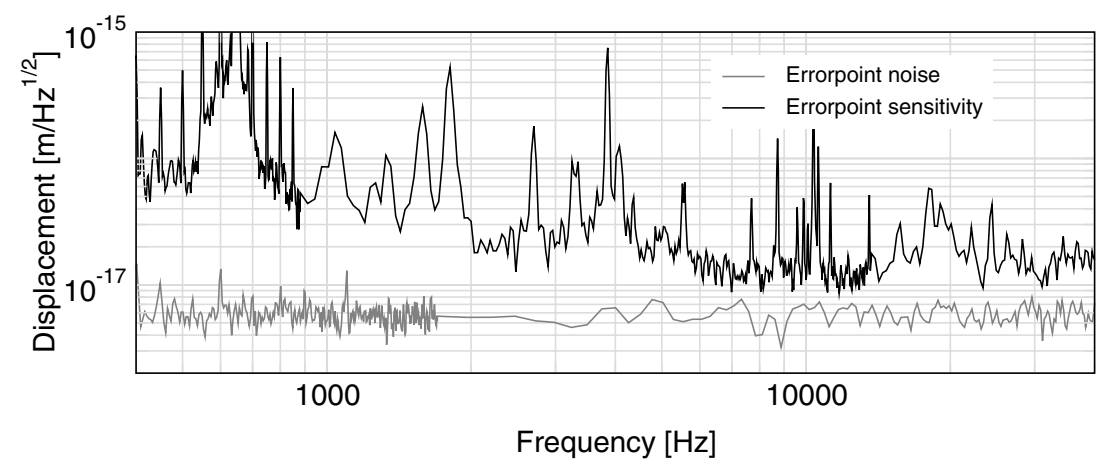

Figure 6. The sensitivity at high frequencies measured at the error point is limited by mechanical resonances and electronic noise.

of 1000 ppm, a better signal to noise ratio at the photo diode and the mixer was achieved. This improved the sensitivity in the $\mathrm{kHz}$ region (see figure 6).

Several noise sources have been identified and addressed; however, the sensitivity achieved does not yet allow the measurement of the pendulum thermal noise. In the future additional changes to the suspension system and a better isolation of the optical components from acoustic excitation by placing them into the vacuum system could reduce the limiting noise sources.

\section{Acknowledgment}

This work was supported by the Deutsche Forschungsgemeinschaft within the Sonderforschungsbereich 407.

\section{References}

[1] Numata K, Otsuka S, Ando M and Tsubono K 2002 Class. Quantum Grav. 191697

[2] Black E 2002 http://www. ligo. caltech. edu/docs/G/G020460-00/

[3] Bracci L et al 2002 Class. Quantum Grav. 191675

[4] Slagmolen B J J, Brokmann X, Shaddock D A, Gray M B and McClelland D E 2002 Class. Quantum Grav. 19 1683

[5] Leonhardt V, Ribichini L, Klövekorn P, Willke B, Lück H and Danzmann K 2002 Class. Quantum Grav. 191717 